ment to the membrane on which it rests. Gradually it increases in size, and, undergoing the changes before explained, becomes firmer, cartilaginous, or even osseous in the centre, and at the same time polished on the exterior. While it increases in size and undergoes these changes of structure, it projects more into the cavity of the joint, and its attachment to the synovial membrane is diminished. In this way it becomes pediculated like a polypus. By degrees the pedicle decreases in thickness, and at last gives way or is broken during some of the movements of the joint. You may sometimes see upon the surface of the loose cartilage, in a rough spot or cicatrix-like appearance, the trace of the point at which this connection existed. The mass is now loose in the articular cavity, having no connection by vessels or other tissue with any part of the body. Still it may derive the elements for its nutrition by imbibition from the synovial fluid in which it floats; and there can be little doubt that the growth of the foreign body, as well as the changes in its interior, continue after it has been separated from its point of attachment.

The evidences of an inflammatory origin and of similar changes are stronger in the case of the melon-seed-like bodies occasionally found in bursæ. These bodies are usually preceded and accompanied by symptoms of inflammation, and they are met with in various stages of their progress. In the same bursa you may see some of them attached by a broad surface to the lining membrane, others pediculated or recently separated, and some smoothed down and deprived of all trace of their former connection.

Supposing this view to be correct, the loose cartilages in joints and the melon-seed-like bodies in bursæ are probably of the same nature with the white spots so often found upon the pericardium and the dense white masses of less frequent, though not uncommon, occurrence upon the spleen and liver. They all appear to originate in deposits of lymph, modified according to the particular circumstances in which they are placed. It is true that the attendant inflammatory symptoms are not always, or indeed generally, present ; but, inasmuch as these substances consist of that which is one of the ordinary products of inflammation, and inasmuch as they are often accompanied by other evidences of inflammatory disturbance, we cannot err very much in this opinion of their nature. Many other morbid products of the same kind may be mentioned, such as the white plates upon the arachnoid, the cicatrices upon the pleura so repeatedly met with near the apices of the lungs, the adhesion between the opposed pleural and pericardial surfaces, the little masses in the subcutaneous cellular tissue which slowly suppurate and burst or gradually disappear, and the corresponding lymph formations in the substance of the lungs and other internal organs, of which various specimens are continually presenting themselves in the dead room at the Hospital. In all, or nearly all of these, the appearance of the morbid product is the first symptom of the disease; and it is only by comparing them with others of the same class, and watching their progress, that we can form an opinion respecting their real character.
Although we state generally that these substances are of inflammatory nature, there can be no question that many of them, such as loose cartilages and the opaque masses on the spleen, possess a power of growth independent of any accompanying inflammation. In some the first formation may have been due to an inflammatory disturbance, but they go on increasing long after that exciting cause has ceased to exist, and in others there is no trace of its existence either in their incipient or their subsequent stages. We cannot fail to remark the similarity in this respect to the origin and progress of warts, polypi, and the other growths of that nature which we have been discussing.

I have alluded to these lymph-formations for the purpose of explaining to you that it is not the natural tissues only which are the subject of this slowly progressive hypertrophic enlargement or growth, inasmuch as certain morbid products, more or less clearly referable to inflammation, exhibit a similar disposition under corresponding circumstances. To this point we must again revert when we enter upon the subject of "tumours," strictly so called.

It may be remarked, in conclusion, that organs in a state of general enlargement or hypertrophy, are, as might be expected, particularly liable to hypertrophy in a circumscribed form. This femur, for instance, is in the whole length of its shaft, thickened to twice its natural size by inflammation, and there is a distinct and considerable exostosis above its inner condyle. The enlarged thyroid gland is often the seat of cystic formations in some part of it; and the enlarged prostate is particularly likely to be deformed by the projection of certain portions, more hypertrophied than the rest, into the cavity of the bladder.

\section{CASE OF UTERINE POLYPUS}

COEXISTING WITH PREGNANCY AND SPONTANEOUS EXPULSION OF THE TUMOUR.

By H. LleWEllyn Williams, M.D., Edin.,

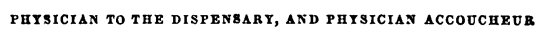

ThE following case, recently occurring under my observation, will be perused with interest, in consequence of the increased attention which has of late been directed to the study of uterine diseases.

Jemima $\mathrm{S} \longrightarrow$, aged 26 , residing in the country, having had one child previously, was on the 20th of March delivered, by a widwife, of a fine female child. The woman who attended her described the case as one of unusual delay, for though the pains were strong and continuous, for some time the head seemed to make no advance. At last the child was born, the placenta being expelled shortly afterwards. She continued to progress favourably until the evening of the 22nd, when she was attacked by rigors, attended with considerable pain immediately above the pubes, and entire cessation of the lochia. On the morning of the 23rd, when I first 
visited her, $\mathbf{I}$ found her in intense suffering, the pain during the night having become gradually diffused over the whole surface of the abdomen; she was harassed by constant nausea and incessant thirst; the abdomen considerably distended with flatus; pulse 130, small and wiry; countenance anxious; breathing short and hurried ; tongue coated and bilious. She was immediately bled to twenty ounces, and hot flannels soaked with turpentine applied to her. body, and a pill, composed of one grain of calomel, and half a grain of opium, administered every three hours, under the impression that this was a regular case of puerperal peritonitis.

The next day $I$ found her somewhat relieved, and ordered the remedies to be persevered with. On the $25 \mathrm{th}$, in consequence of her complaining of a good deal of uneasiness above the pubes, she was ordered a blister, to be dressed with mercurial ointment, with a view of ensuring its more rapid absorption. She continued to improve daily, and by the $28 \mathrm{th}$, the eighth day after her confinement, I considered her rapidly convalescing; but on the evening of that day, she complained a good deal of pain in the loins, shooting through to the uterus, which, however, did not prevent her sleeping. Whilst making water, early on the morning of the 29th, she was attacked with violent flooding, attended by bearing-down pains. In the course of half an hour a large mass was expelled, with increased hæmorrhage which induced syncope to such an alarming extent that the people in attendance on her thought death had ensued, and it was some time before she rallied. On being summoned, I examined the substance, which proved to be a polypus, or pediculated tumour, with an elongated neck, the entire mass weighing between three and four ounces. The hæmorrhage was easily controlled, by the use of astringents and local cold applications. No further loss of blood occurred; my patient steadily gained strength, and was shortly quite recovered.

Remarks. - This case is worthy of notice, from the entire absence of any of those symptoms-hæmorrhage, leucorrhœa, \&c.-marking the existence of uterine disease. The entire freedom from these affections is contrary to the ordinary symptoms noticed by systematic writers, who assert that polypi and all tumours of the uterus are accompanied by more or less hæmorrhage, proceeding according to some from the lacerated portion of the pedicle, but much more likely as an exudation from the mucous surface of the tumour itself. My patient, however, never complained-never noticed anything unusual, either before or during the period of pregnancy.

The correct diagnosis of the presence of fibrous tumours and polypi before they have been forced into the vagina has been a matter of much uncertainty. Dupuytren* remarks:- "When polypi are entirely included within the uterus, the rational symptoms afford room for conjecture only, and examination by

* Leçons orales, vol. iii., p. 490 the finger or speculum are alike insufficient." Roche and Sanson* say there is but one case in which a certain diagnosis may be formed,-viz., when the neck being effaced and partly opened, it is possible to feel the rounded tumour within." Some of the older pathologists seem to have thought that no danger was to be apprehended so long as the mass was within the uterus. It is only very recently that Dr. Simpson, of Edinburgh, to remove these difficulties, has directed the attention of the profession to the capability of dilating the os and cervix uteri, by means of sponge tents, thereby rendering a correct diagnosis much more readily attainable. It is also interesting to note how the ars medicina was supplanted by the vis naturce. The specific inflammation commencing in the neck of the tumour during parturition, extending to the adjacent part of the uterus, and from thence to the peritoneum, gave rise undoubtedly to those symptoms which I was first called upon to treat, and which led to the belief of its being a case of puerperal peritonitis.

The subsequent ulceration of the attachments of the tumour, hæmorrhàge, and disengagement of the mass, revealed the true nature of the disease, which would otherwise have been set down as a successful case of puerperal peritonitis.

Dr. Merryman, in his essay on this subject, relates cases, where the impeding mass having been torn from its attachments was expelled in the act of parturition before the head. Such examples are not unfrequent; but instances such as I have just detailed of the patient, after having escaped the peril of child-birth, being exposed to the fresh danger of inflammation of the uterine parietes, and spontaneous expulsion of the mass, are extremely rare.

\section{ON}

\section{TRAUMATIC TETANUS.}

\section{By GEORGE SAYLE, Esa.,}

SORGEON TO THE WHBT NORPOLE AND IYMX HOSPITAL, PORMERLY CURATOR OF THB ANATOMICAL MUSEUM IN THE ST. GROBgr's HOSPITAL SCHOOL.

The following observations have their origin in a request contained in the last number of the Provincial Medical and Surgical Journal, from my colleague Dr. Whiting, relative to a case of trismus, occurring in the West Norfolk and Lynn Hospital, under my care. It is not in my power to give a full report of the case, from the notes being accidentally mislaid, nor should I have done so had it been otherwise, as I do not think there was sufficient in it, either in symptoms or treatment, upon which anything could have been said, in addition to the many conflicting statements already extant. His report, so far as it goes, is quite correct, and under the treatment a successful termination ensued. The man was admitted for a machine accident, by which the inner part of the left foot was so much destroyed,

\footnotetext{
* Noreaux Elèmens de Patbol. Med. Chir., tome iii., p. 284.
} 